Pacific Journal of Mathematics

ENDOMORPHISM RINGS OF FINITELY GENERATED 


\title{
ENDOMORPHISM RINGS OF FINITELY GENERATED PROJECTIVE MODULES
}

\author{
ROBERT W. MiLLER
}

\begin{abstract}
Over a ring $A$ let $P_{A}$ be a finitely generated projective right $A$-module with $A$-endomorphism ring $B$. Anderson has called $P_{A}$ an injector, perfect injector, projector, perfect projector, if the functor $F={ }_{B} P \otimes_{A}$ ( ) preserves injectives, injective hulls, projectives, projective covers, respectively. Call $P_{A}$ a flatjector if $F$ preserves flat modules. Injectors, flatjectors, and projectors are characterized. The radical of a module over $B$ is studied, and necessary and sufficient conditions are given for the radical of $B$ to be left $T$-nilpotent. Perfect injectors are characterized. Previous characterizations of perfect projectors have assummed the ring $A$ to be left perfect. Here characterizations are obtained using substantially weaker conditions on $P_{A}$.
\end{abstract}

Over a ring $A$ let $P_{A}$ be a finitely generated projective right $A$ module with $A$-endomorphism ring $B$. Let $T$ be the trace of $P_{A}$ and $P^{*}=\operatorname{Hom}_{A}(P, A)$ be the $A$-dual of $P$. Injectors, flatjectors, and projectors are characterized in $\S 2$, the characterizations of injectors and projectors being extensions of results due to Anderson [1]. For example, $P_{A}$ is a flatjector if and only if ${ }_{B} P$ (equivalently ${ }_{A} P^{*} \otimes{ }_{B} P$ ) is flat. $P_{A}$ is a projector if and only if the natural map $\eta:{ }_{A} P^{*} \otimes P \rightarrow$ ${ }_{A} T$ is a projective cover. It is shown that $P_{A}$ is a flatjector if and only if ${ }_{A} P^{*}$ is an injector. Furthermore, every projector is a flatjector, the two being equivalent for perfect rings. Examples are given of a flatjector that is not a projector and a nonperfect ring where every flatjector is a projector.

In $\S 3$ it is shown that the $B$-radical of ${ }_{B} P \otimes{ }_{A} X$ is isomorphic to ${ }_{B} P \otimes{ }_{A} J(T X)$. In particular the $B$-radical of ${ }_{B} P$ is isomorphic to ${ }_{B} P \otimes{ }_{A} J(T)$. A definition of $U$-dominant codimension dual to the definition of $V$-dominant dimension given in [8] is introduced. The radical $N$ of $B$ being left $T$-nilpotent is characterized in terms of the full subcategories $\mathscr{C}_{1}\left({ }_{A} P^{*}\right)$ of ${ }_{A} \mathfrak{M}$ consisting of all left $A$-modules of ${ }_{A} P^{*}$-dominant codimension $\geqq 1$, and $\mathfrak{D}_{1}\left(Q_{A}\right)$ of $\mathfrak{M}_{A}$ consisting of all right $A$-modules of $Q_{A}$-dominant dimension $\geqq 1$ (where $Q_{A}=\operatorname{Hom}_{B}(P, W)$ for ${ }_{B} W$ an injective cogenerator). It is shown that $N$ is left $T$-nilpotent if and only if $J T$ is left $T$-nilpotent, or equivalently $J\left(\oplus_{I} P^{*}\right)$ is small in $\bigoplus_{I} P^{*}$ for any index set $I$.

In $\S 4$ perfect injectors are characterized in terms of their trace ideal and certain conditions on large submodules. Anderson [1] has studied perfect projectors when the ring $A$ is left perfect. Here 
characterizations of perfect projectors are obtained using substantially weaker conditions on $P_{A}$, e.g. $J T$ being left $T$-nilpotent. Finally it is shown that for $A$ semiperfect, $P_{A}$ is a perfect projector if and only if ${ }_{A} P^{*}$ is a perfect injector.

1. Preliminaries. Throughout this paper we will observe the following definitions and notation. $A$ will be a ring with unit and all modules will be unitary. All maps will be written on the side opposite the scalars. Given an $A$-module $X$, its $A$-dual $\operatorname{Hom}_{A}(X, A)$ will be denoted by $X^{*} . \quad P_{A}$ will always be a finitely generated projective right $A$-module with $A$-endomorphism ring $B$. It is easy to see that ${ }_{A} P^{*}$ is also finitely generated projective and that $\left(P^{*}\right)^{*} \cong P$. With $P_{A}$ we associate the two-side ideal $T$ of $A$ (called the trace of $P_{A}$ ) generated by the images of all $A$-homomorphisms from $P$ into $A$, i.e., $T=\sum_{f \in P^{*}} \operatorname{im} f$.

Let ${ }_{A} \mathfrak{M}\left(\mathfrak{M}_{A}\right)$ represent the category of left (right) $A$-modules. We have functors

$$
\begin{aligned}
& F={ }_{B} P \otimes{ }_{A}():{ }_{A} \mathfrak{M} \rightarrow{ }_{B} \mathfrak{M} \\
& G={ }_{A} P^{*} \otimes{ }_{B}():{ }_{B} \mathfrak{M} \rightarrow{ }_{A} \mathfrak{M}
\end{aligned}
$$

and associated natural transformations

$$
\begin{gathered}
\eta_{X}: G F\left({ }_{A} X\right) \rightarrow{ }_{A} X \\
\mu_{W}: F G\left({ }_{B} W\right) \rightarrow{ }_{B} W
\end{gathered}
$$

defined by

$$
\begin{gathered}
(f \otimes p \otimes x) \eta_{X}=f(p) x \\
(p \otimes f \otimes w) \mu_{W}=p f() w
\end{gathered}
$$

for $p \in P, f \in P^{*}, x \in X$, and $w \in W$.

The following isomorphisms are well known (see e.g. [4]).

I. For ${ }_{A} X,{ }_{B} U_{A},{ }_{B} W$

$$
\operatorname{Hom}_{B}\left({ }_{B} U \otimes{ }_{A} X,{ }_{B} W\right) \cong \operatorname{Hom}_{A}\left({ }_{A} X,{ }_{A} \operatorname{Hom}_{B}\left({ }_{B} U_{A},{ }_{B} W\right)\right)
$$

II. For $U_{A},{ }_{B} M_{A},{ }_{B} W$ where $U_{A}$ is finitely generated projective

$$
U \otimes{ }_{A} \operatorname{Hom}_{B}\left({ }_{B} M_{A},{ }_{B} W\right) \cong \operatorname{Hom}_{B}\left({ }_{B} \operatorname{Hom}_{A}\left(U_{A},{ }_{B} M_{A}\right),{ }_{B} W\right)
$$

Properties of $P, P^{*}, T$, and $B$.

(a) $T^{2}=T, P T=P$, and $T P^{*}=P^{*}$.

(b) $B=$ End $\left({ }_{A} P^{*}\right)$.

(c) ${ }_{B} P \otimes{ }_{A} T_{A} \cong{ }_{B} P_{A}$ and ${ }_{A} T \otimes{ }_{A} P_{B}^{*} \cong{ }_{A} P_{B}^{*}$.

(d) $\operatorname{im} \eta_{X}=T X$ for all $X \in \in_{A} \mathfrak{M}$.

(e) $F G$ is naturally equivalent to the identity functor on 
${ }_{B} \mathfrak{M}\left(F G \sim I_{B^{\mathfrak{N}}}\right)$ via $\mu$.

(f) ${ }_{B} P \otimes{ }_{A} T X \cong{ }_{B} P \otimes{ }_{A} X$ and $Y T \otimes{ }_{A} P_{B}^{*} \cong Y \otimes_{A} P_{B}^{*}$.

(g) ${ }_{B} P \otimes{ }_{A} X \cong{ }_{B} \mathrm{Hom}_{A}\left({ }_{A} P_{B}^{*},{ }_{A} X\right)$ and $Y \otimes{ }_{A} P_{B}^{*} \cong \operatorname{Hom}_{A}\left({ }_{B} P_{A}, Y_{A}\right)_{B}$.

(h) For $X \in{ }_{A} \mathfrak{M}, P \otimes{ }_{A} X=0$ if and only if $T X=0$.

(i) For $Y \in \mathfrak{M}_{A}, \operatorname{Hom}_{A}(P, Y)=0$ if and only if $Y T=0$.

Proof. (a), (c), (d), and (e) are essentially in [1].

(b) See [10, Lemma 1.1].

(f) ${ }_{B} P \otimes{ }_{A} X / T X \cong{ }_{B} P \otimes{ }_{A} A / T \otimes X \cong P / P T \otimes{ }_{A} X=0$ by (a). The second isomorphism follows similarly.

(g) follows easily from II.

(h) follows easily from (d) and (f).

(i) follows easily from (f), (g), and the dual of (d).

Let $K$ be a submodule of $X$. We say $K$ is small in $X$ (and write $\left.K^{\prime} \subseteq X\right)$ if given a submodule $H$ of $X$ such that $K+H=X$, then $H=X$. We say $K$ is large in $X$ (and write $K \subseteq^{\prime} X$ ) if $K$ has nonzero intersection with every nonzero submodule of $X$.

For an $A$-module $X$ the radical of $X$, denoted $J(X)$, is defined to be the intersection of all maximal submodules of $X$. If $X$ has no maximal submodules, then $J(X)=X$ (e.g. see [7]). We will write $J$ for $J(A)$.

The following properties of the radical are well known. Properties of the radical: For ${ }_{A} X$,

(a) If $x \in J(X)$, then $A x^{\prime} \subseteq X$.

(b) If $K^{\prime} \subseteq X$, then $K \subseteq J(X)$.

(c) If $f: X \rightarrow Y$ is an $A$-homomorphism, then $(J(X)) f \subseteq J(Y)$.

(d) $J X \subseteq J(X)$.

(e) If $X$ is projective, then $J X=J(X)$. [3]

(f) If $X$ is projective, then $J X \neq X$. [3]

Notation. To minimize confusion in later theorems, the radical of a module $W$ over the ring $B=$ End $\left(P_{A}\right)$ will be denoted by $N(W)$. In particular, $N=N(B)$.

Lemma 1.1. Let $f:{ }_{A} X \rightarrow{ }_{A} Y$ be a projective cover. Then $J(Y)=$ $J Y$.

Proof. Since $J Y \subseteq J(Y)$ always, we only need to show that $J(Y) \subseteq$ $J Y$. Let $y \in J(Y)$. Then $y$ is contained in every maximal submodule of $Y$. Thus $(y) f^{-1}$ is contained in every maximal submodule of $X$ containing $K=\operatorname{ker} f$. But $K^{\prime} \subseteq X$ and hence is contained in every maximal submodule of $X$. Thus $(y) f^{-1} \in J(X)=J X$. So $y \in(J X) f=$ $J(X) f=J Y$. 
LEMMA 1.2. If a flat module ${ }_{A} Y$ has a projective cover, then ${ }_{A} Y$ is projective.

Proof. Let $f:{ }_{A} X \rightarrow{ }_{A} Y$ be a projective cover of ${ }_{A} Y$. We have the exact sequence

$$
0 \longrightarrow K \longrightarrow X \stackrel{f}{\longrightarrow} Y \longrightarrow 0
$$

where $K=\operatorname{ker} f$. Let $k \in K$. By [11, Lemma 2.2] there exists $\theta_{k}: X \rightarrow$ $K$ such that $(k) \theta_{k}=k$. But $\operatorname{im} \theta_{k} \subseteq K^{\prime} \subseteq X$, hence $\theta_{k}$ is contained in the radical of the endomorphism ring of ${ }_{A} X$ (see [11, Prop. 1.1]). So $1-\theta_{k}$ is a unit (see e.g. [7]). Therefore, $(k)\left(1-\theta_{k}\right)=k-(k) \theta_{k}=0$. Hence $k=0$. Thus ${ }_{A} Y$ is projective since $f$ is an isomorphism.

Notation. $X^{I}$ will denote the direct sum of copies of $X$ over the index set $I$. If $I=\{1, \cdots, n\}$, we will simply write $X^{n}$ for $X^{I}$.

Let ${ }_{A} Y \subseteq{ }_{A} X$ and let $L$ be a right ideal of $A$. Then $(Y: L)_{X}=$ $\{x \in X \mid L x \subseteq Y\}$ is a submodule of $X$ containing $Y$. In particular $r_{X}(L)=(0: L)_{X}$ is called the right annihilator of $L$ in $X$. If $Y_{A} \subseteq X_{A}$ and $L$ is a left ideal of $A$ similar definitions apply. We will write $l_{X}(L)$ for the left annihilator of $L$ in $X$.

The following lemmas are left to the reader.

Lemma 1.3. Let $L$ be a two-sided ideal of $A$. Then

(a) For ${ }_{A} X, r_{X}(L) \cong \operatorname{Hom}_{A}(A / L, X)$.

(b) For $X_{A}, l_{X}(L) \cong \operatorname{Hom}_{A}(A / L, X)$.

(c) $r_{A}(L)$ and $l_{A}(L)$ are both two-sided ideals of $A$.

LemMA 1.4. Let ${ }_{B} U_{A}$ be a bimodule with ${ }_{B} U$ flat. If ${ }_{A} X$ is flat, then ${ }_{B} U \otimes{ }_{A} X$ is flat.

2. Injectors, flatjectors, and projectors. For a finitely generated projective right $A$-module $P_{A}$ with $A$-endomorphism ring $B$, Anderson [1] has called $P_{A}$ an injector (projector) if the functor $F=$ ${ }_{B} P \otimes{ }_{A}()$ preserves injectives (projectives). That is, if ${ }_{A} X$ is injective (projective) in ${ }_{A} \mathfrak{M}$, then ${ }_{B} P \otimes{ }_{A} X$ is injective (projective) in ${ }_{B} \mathfrak{M}$. Similarly, we call $P_{A}$ a flatjector if the functor $F={ }_{B} P \otimes_{A}()$ preserves flat modules. Our purpose in this section is to characterize injectors, flatjectors, and projectors.

THEOREM 2.1. For $P_{A}$ finitely generated projective the following statements are equivalent.

(a) $P_{A}$ is an injector.

(b) $P_{B}^{*}$ is flat in $\mathfrak{M}_{B}$. 
(c) $P^{*} \otimes{ }_{B} P_{A}$ is flat in $\mathfrak{M}_{A}$.

Proof. $\quad(a) \Leftrightarrow(b)$. This is by Anderson [1, Theorem 2.1].

(b) $\Rightarrow$ (c). Since both $P_{B}^{*}$ and $P_{A}$ are flat (c) follows by Lemma 1.4. (c) $\Rightarrow(b)$. Let $\alpha:{ }_{B} U \rightarrow{ }_{B} V$ be a monomorphism and consider the exact sequence

$$
0 \longrightarrow K \longrightarrow G(U) \stackrel{G(\alpha)}{\longrightarrow} G(V)
$$

where $K=\operatorname{ker} G(\alpha)$. Since $P_{A}$ is flat

$$
0 \longrightarrow F(K) \longrightarrow F G(U) \longrightarrow F G(V)
$$

is exact. Thus $F(K)=0$ since $F G \sim I_{B^{\mathfrak{m}}}$. Since $P^{*} \otimes{ }_{B} P_{A}$ is flat

$$
0 \longrightarrow G F(K) \longrightarrow G F G(U) \longrightarrow G F G(V)
$$

is exact. Thus $P_{B}^{*}$ is flat since $G F(K)=0$ and $F G \sim I_{B}$.

We include the following theorem for completeness. With the obvious changes, the proof is similar to the proof of $(c) \Rightarrow(b)$ in Theorem 2.1.

TheOREM 2.2. (Anderson [1, Theorem 2.2]). If the trace ideal $T$ of $P_{A}$ is flat in $\mathfrak{M}_{A}$, then $P_{A}$ is an injector.

The converse to Theorem 2.2 is false as shown by [1, Example 2.3].

For flatjectors we give a characterization similar to the one given for injectors.

THEOREM 2.3. For $P_{A}$ finitely generated projective the following statements are equivalent.

(a) $P_{A}$ is a flatjector.

(b) ${ }_{B} P$ is flat in ${ }_{B} \mathfrak{M}$.

(c) ${ }_{A} P^{*} \otimes{ }_{B} P$ is flat in ${ }_{A} \mathfrak{M}$.

Proof. $\quad(\mathrm{a}) \Longrightarrow(\mathrm{b})$. Since ${ }_{A} A$ is flat and ${ }_{B} P \cong{ }_{B} P \otimes{ }_{A} A$, we must have ${ }_{B} P$ flat.

$(b) \Rightarrow(c)$. Both ${ }_{B} P$ and ${ }_{A} P^{*}$ are flat. Thus (c) follows from Lemma 1.4.

(c) $\Rightarrow$ (a). Let $\alpha: U_{B} \rightarrow V_{B}$ be a monomorphism.

Let $F^{*}$ and $G^{*}$ represent the functors ()$\otimes{ }_{A} P_{B}^{*}$ and ()$\otimes{ }_{B} P_{A}$ respectively. Consider the exact sequence

$$
0 \longrightarrow K \longrightarrow G^{*}(U) \stackrel{G^{*}(\alpha)}{\longrightarrow} G^{*}(V)
$$


where $K=\operatorname{ker} G^{*}(\alpha)$. Since ${ }_{A} P^{*}$ is flat

$$
0 \longrightarrow F^{*}(K) \longrightarrow F^{*} G^{*}(U) \longrightarrow F^{*} G^{*}(V)
$$

is exact. Thus $F^{*}(K)=0$ since $F^{*} G^{*} \sim I_{\mathfrak{M}_{B}}$. Since ${ }_{A} P^{*} \otimes{ }_{B} P$ is flat

$$
0 \longrightarrow G^{*} F^{*}(K) \longrightarrow G^{*} F^{*} G^{*}(U) \longrightarrow G^{*} F^{*} G^{*}(V)
$$

is exact. Thus ${ }_{B} P$ is flat since $G^{*} F^{*}(K)=0$ and $F^{*} G^{*} \sim I_{\mathfrak{n}_{B}}$.

Let ${ }_{A} X$ be flat. Then ${ }_{B} P \otimes{ }_{A} X$ is flat by Lemma 1.4. So $P_{A}$ is a flatjector.

Theorems 2.1 and 2.3 show that injector and flatjector are dual properties.

Corollary 2.4. For a finitely generated projective module $P_{A}$, $P_{A}$ is an injector if and only if ${ }_{A} P^{*}$ is a flatjector.

A ring $A$ is said to be regular if every (right) $A$-module is flat. Ware [11] has called a projective module regular if every homomorphic image is flat. In the following corollary we give a new proof to one of Ware's results.

Corollary 2.5. (Ware [11, Theorem 3.6]). Let $P_{A}$ be a finitely generated regular module with $B=$ End $\left(P_{A}\right)$. Then $B$ is a regular ring.

Proof. We first note that $P_{A}^{I}$ is a regular module for any index set $I$ [11, page 239]. Let $U_{B} \in \mathfrak{M}_{B}$ and consider $U \otimes_{B} P_{A}$. Let $I=U$ and define a map $\varphi: P_{A}^{I} \rightarrow U \otimes{ }_{B} P_{A}$ by $\varphi\left[\left(p_{u}\right)_{u \in I}\right]=\Sigma u \otimes p_{u}$. Clearly $\varphi$ is an $A$-epimorphism. Thus $U \otimes{ }_{B} P_{A}$ is flat in $\mathfrak{M}_{A}$.

In particular $P^{*} \otimes{ }_{B} P_{A}$ is flat in $\mathfrak{M}_{A}$. Thus ${ }_{A} P^{*}$ is a flatjector by Theorem 2.3. Hence $U_{B} \cong U \otimes{ }_{B} P \otimes{ }_{A} P_{B}^{*}$ is flat in $\mathfrak{M}_{B}$. So $B$ is a regular ring.

Theorem 2.6. If the trace ideal $T$ of $P_{A}$ is flat in ${ }_{A} \mathfrak{M}$, then $P_{A}$ is a flatjector.

With the obvious changes, Theorem 2.6 follows in the same manner as (c) $\Rightarrow$ (a) of Theorem 2.3. Hence the proof will be omitted. The converse of Theorem 2.6 is false. In Example 2.3 of [1], $P_{A}$ is a flatjector, but $T$ is not flat in ${ }_{A} \mathfrak{M}$.

Before giving a characterization for projectors, we need the following lemma.

Lemma 2.7. The map $\eta_{A}: P^{*} \otimes_{B} P \rightarrow T$ is both a right and left 
minimal A-epimorphism.

Proof. We will only prove the left case as the right case follows by symmetry.

Suppose we have $\alpha:{ }_{A} X \rightarrow{ }_{A} P^{*} \otimes{ }_{B} P$ such that $\alpha \eta_{A}$ is onto. Tensoring with $P_{A}$ we have

$$
F(X) \stackrel{F(\alpha)}{\longrightarrow} F\left(P^{*} \otimes P\right) \stackrel{F\left(\eta_{A}\right)}{\longrightarrow} F(T)
$$

where $F(\alpha) F\left(\eta_{A}\right)$ is onto. Since $F\left(P^{*} \otimes P\right)=F G(P) \cong P \cong F(T), F\left(\eta_{A}\right)$ is an isomorphism. Thus $F(\alpha)$ is onto. So

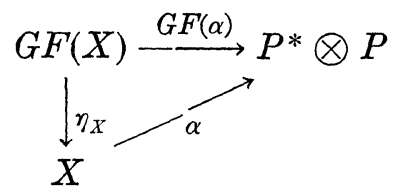

is a commutative diagram where $G F(\alpha)$ is onto. Hence, $\alpha$ is onto.

THEOREM 2.8. For $P_{A}$ finitely generated projective the following statements are equivalent.

(a) $P_{A}$ is a projector.

(b) ${ }_{B} P$ is projective in ${ }_{B} \mathfrak{M}$.

(c) ${ }_{A} P^{*} \otimes{ }_{B} P$ is projective ${ }_{A} \mathfrak{M}$.

(d) $\eta_{A}:{ }_{A} P^{*} \otimes P \rightarrow{ }_{A} T$ is a projective cover for ${ }_{A} T$.

Proof. (a) $\Leftrightarrow(\mathrm{b})$. This is by Anderson [1, Theorem 3.1]. $(b) \Rightarrow(c)$. Since ${ }_{B} P$ is projective we have a split exact sequence

$$
{ }_{B} B^{I} \rightleftarrows{ }_{B} P \longrightarrow 0
$$

where $I$ is some index set. Thus

$$
G\left(B^{I}\right) \rightleftarrows G(P) \longrightarrow 0
$$

is also split exact. Since $G\left(B^{I}\right) \cong{ }_{A} P^{* I}$, we see that $G(P)={ }_{A} P^{*} \otimes{ }_{B} P$ is a direct summand of a projective, and hence is projective.

(c) $\Rightarrow(d)$. This is immediate from Lemma 2.7.

$(\mathrm{d}) \Rightarrow(\mathrm{b})$. As in Corollary 2.5, ${ }_{A} P^{*} \otimes{ }_{B} P$ is the homomorphic image of a direct sum of copies of ${ }_{A} P^{*}$, say ${ }_{A} P^{* I}$. Since ${ }_{A} P^{*} \otimes{ }_{B} P$ is projective, we have

$$
{ }_{A} P^{* I} \rightleftarrows{ }_{A} P^{*} \otimes{ }_{B} P \longrightarrow 0
$$

is split exact. Tensoring with $P_{A}$, we see that ${ }_{B} P$ is a direct summand of a direct sum of copies of ${ }_{B} B$. Thus ${ }_{B} P$ is projective.

The following theorem is added for completeness. With the 
obvious changes, the proof is similar to the proof of $(d) \Rightarrow(b)$ of Theorem 2.8.

Theorem 2.9. (Anderson [1, Theorem 3.2]). If the trace ideal $T$ of $P_{A}$ is projective in ${ }_{A} \mathfrak{M}$, then $P_{A}$ is a projector.

The converse to Theorem 2.9 is false as shown by [1, Example 2.3].

One might ask when the functor $F={ }_{B} P \otimes{ }_{A}()$ preserves finitely generated projectives? That is, if ${ }_{A} X$ is finitely generated projective, when is ${ }_{B} P \otimes{ }_{A} X$ finitely generated projective? This question is answered by the following theorem.

THEOREM 2.10. For $P_{A}$ finitely generated projective the following statements are equivalent.

(a) $F={ }_{B} P \otimes{ }_{A}($ ) preserves finitely generated projectives.

(b) ${ }_{B} P$ is finitely generated projective in ${ }_{B} \mathfrak{M}$.

(c) ${ }_{B} P^{*} \otimes{ }_{B} P$ is finitely generated projective in ${ }_{A} \mathfrak{M}$.

Proof. $\quad(a) \Rightarrow(b)$. Since ${ }_{i 1} A$ is finitely generated projective, so is ${ }_{B} P \cong{ }_{B} P \otimes A$.

$(b) \Rightarrow(c)$. Since ${ }_{B} P$ is finitely generated, the index set $I$ used in $(\mathrm{b}) \Rightarrow(\mathrm{c})$ of Theorem 2.8 can be taken to be finite. Thus ${ }_{A} P^{*} \otimes{ }_{B} P$ is finited generated projective.

$(c) \Rightarrow(b)$. Again the index set $I$ used in $(d) \Rightarrow(b)$ of Theorem 2.8 can be taken to be finite. Thus ${ }_{B} P$ is finitely generated projective.

(b) $\Rightarrow$ (a). If ${ }_{A} X$ is finitely generated projective, then there is a split exact sequence

$$
{ }_{4} A^{n} \rightleftarrows{ }_{A} X \longrightarrow 0 \text {. }
$$

Thus tensoring with $P_{A}$ we see that ${ }_{B} P \otimes{ }_{A} X$ is a direct summand fo ${ }_{B} P \otimes{ }_{A} A^{n} \cong\left({ }_{B} P \otimes{ }_{A} A\right)^{n} \cong{ }_{B} P^{n}$. Since ${ }_{B} P^{n}$ is finitely generated projective, so is ${ }_{B} P \otimes{ }_{A} X$.

THEOREM 2.11. If the trace ideal $T$ of $P_{A}$ is finitely generated projective in ${ }_{A} \mathfrak{M}$, then $F={ }_{B} P \otimes_{A}()$ preserves finitely generated projectives.

Proof. By Theorem 2.9, $P_{A}$ is a projector, hence ${ }_{B} P$ is projective. By [10, Theorem 2.2, Corollary 4] ${ }_{B} P$ is finitely generated. Hence the theorem follows by Theorem 2.10.

REMARK. By Theorems 2.3 and 2.8 we see that every projector 
is a flatjector. On page 328 of [1] Anderson gives an example of a projector (hence flatjector) that is not an injector. Also, Anderson constructs an injector that is not flat over its endomorphism ring, and thus is neither a flatjector nor a projector.

In [3] Bass defines a ring $A$ to be left (right) perfect if every left (right) $A$-module has a projective cover, and semiperfect if every cyclic (left) $A$-module has a projective cover. Bass shows that $A$ is left perfect if and only if evey flat left $A$-module is projective. This is also easily seen from Lemma 1.2.

By Theorems 2.3 and 2.8, for a left perfect ring $A, P_{A}$ is a flatjector if and only if $P_{A}$ is a projector. The question arises as to whether a left perfect ring can be characterized in terms of every flatjector being a projector. We exhibit an example to show that the answer to this question is no.

ExAmple 2.12. Let $A$ be a simple ring that is not left perfect. Let $P_{A}$ be a finitely generated projective right $A$-module. Since $A$ is simple, $P_{A}$ is a generator, and hence ${ }_{B} P$ is projective (see [2]). Thus over $A$, every flatjector is a projector, but $A$ is not left perfect.

We end this section with an example of a flatjector that is not a projector.

EXAMPLE 2.13. Let $B$ be a ring that is not left perfect and let ${ }_{B} Q_{C}$ be a $B-C$ bimodule such that ${ }_{B} Q$ is flat, but not projective. Let $A$ be the ring

$$
A=\left(\begin{array}{ll}
B & { }_{B} Q_{C} \\
0 & C
\end{array}\right)
$$

and let

$$
e=\left(\begin{array}{ll}
1 & 0 \\
0 & 0
\end{array}\right)
$$

Then $P_{A}=e A$ is finitely generated projective and End $\left(P_{A}\right)=e A e \cong$ $B$. As a $B$-module, ${ }_{B} P \cong{ }_{B} B \bigoplus_{B} Q$. Thus ${ }_{B} P$ is flat, but ${ }_{B} P$ is not projective. Hence $P_{A}$ is a flatjector but not a projector.

3. Radicals. Our purpose in this section is to answer the following questions. For $P_{A}$ finitely generated projective with $B=$ End $\left(P_{A}\right)$

(1) given ${ }_{A} X$, what is the radical of ${ }_{B} P \otimes{ }_{A} X$ (in particular $\left.{ }_{B} P \cong{ }_{B} P \otimes{ }_{A} A\right)$, and

(2) when is the radical of $B$ left $T$-nilpotent?

Letting $T$ be the trace ideal of $P_{A}$, Sandomierski [10] has defined 
an $A$-module ${ }_{A} X\left(X_{A}\right)$ to be $T$-accessible if $T X=X(X T=X)$. Clearly, both $P_{A}$ and ${ }_{A} P^{*}$ are $T$-accessible.

Lemma 3.1. ${ }_{A} X\left(X_{A}\right)$ is T-accessible if and only if ${ }_{A} X\left(X_{A}\right)$ is the homomorphic image of a direct sum of copies of ${ }_{A} P^{*}\left(P_{A}\right)$.

Proof. We will do the left case only as the right case follows in a similar manner.

Suppose that $X=T X$. Let $I=F(X)$ and define $\varphi: P^{* I} \rightarrow G F(X)$ by $\left[\left(z_{i}\right)_{i \in I}\right] \varphi=\sum z_{i} \otimes i$. Clearly $\varphi$ is an $A$-epimorphism. But via $\eta_{X}$, $T X$ is the homomorphic image of ${ }_{A} P^{* I}$.

Conversely suppose that we have an $A$-epimorphism $\varphi:{ }_{A} P^{* I} \rightarrow{ }_{A} X$ for some index set $I$. Letting $Y=P^{* I}, X=(Y) \varphi=(T Y) \varphi=T(Y) \varphi=$ $T X$.

If ${ }_{B} L \subseteq{ }_{B} \operatorname{Hom}_{A}\left(P^{*}, X\right)$, then ${ }_{A} P^{*} L=\left\{\sum z g \mid z \in P^{*}, g \in L\right\}$ is a $T$ accessible submodule of ${ }_{A} X$. In fact, $P^{*} \operatorname{Hom}_{A}\left(P^{*}, X\right)=T X$.

THEOREM 3.2. (Sandomierski [10, Theorem 2.2]). The correspondence ${ }_{B} L \rightarrow{ }_{A} P^{*} L$ is a one-to-one inclusion preserving correspondence between the submodules ${ }_{B} L$ of ${ }_{B} \mathrm{Hom}_{A}\left(P^{*}, X\right)$ and the T-accessible submodules of ${ }_{A} X$. The inverse correspondence is given by ${ }_{A} Y \rightarrow_{B} \operatorname{Hom}_{A}\left(P^{*}, Y\right)$.

By Theorem 3.2 we see that if ${ }_{A} S$ is a simple $A$-module, then ${ }_{B} \operatorname{Hom}_{A}\left(P^{*}, S\right) \cong{ }_{B} P \otimes{ }_{A} S$ is either zero or a simple $B$-module.

We say that a proper submodule ${ }_{A} Y$ is a maximal $T$-accessible submodule of ${ }_{A} X$ if ${ }_{A} Y$ is $T$-accessible and there are no $T$-accessible submodules strictly between ${ }_{A} Y$ and ${ }_{A} X$. By Theorem 3.2, ${ }_{A} Y$ is a maximal $T$-accessible submodule of $T X$ if and only if ${ }_{B} P \otimes_{A} Y \cong$ ${ }_{B} \mathrm{Hom}_{A}\left(P^{*}, Y\right)$ is a maximal submodule of ${ }_{B} P \otimes{ }_{A} X \cong{ }_{B} \operatorname{Hom}_{A}\left(P^{*}, X\right)$.

THEOREM 3.3. The correspondence $Y \rightarrow(Y: T)_{X}$ is a one-to-one inclusion preserving correspondence between the T-accessible submodules ${ }_{A} Y$ of ${ }_{A} X$ and the submodules ${ }_{A} U$ of ${ }_{A} X$ such that $(T U: T)_{X}=U$. The inverse correspondence is given by $U \rightarrow T U$.

Proof. We show that both composites yield the identity.

(i) Consider $W=(Y: T)_{X}$. Since $Y \subseteq W$ and $T Y=Y$, it is easy to see that $T W=Y$. Hence $(T W: T)_{X}=(Y: T)_{X}=W$. Thus

$$
Y \longrightarrow W \longrightarrow T W=Y \text {. }
$$

(ii) Consider $U$ such that $(T U: T)_{X}=U$. Then

$$
U \longrightarrow T U \longrightarrow(T U: T)_{X}=U \text {. }
$$

One easily checks that the correspondence is inclusion preserving. 
THEOREM 3.4. For ${ }_{4} X$ T-accessible the correspondence defined in Theorem 3.3 yields a one-to-one correspondence between the maximal $T$-accessible submodules of $X$ and the maximal submodules of $X$.

Proof. (i) Let $Y$ be a maximal $T$-accesible submodule of ${ }_{A} X$ and suppose that $(Y: T)_{X} \subseteq V \varsubsetneqq X$. Then $Y=T(Y: T)_{X} \subseteq T V \varsubsetneqq T X=$ $X$. Thus $T V=Y$ so that $V \cong(Y: T)_{X}$. Hence $(Y: T)_{X}$ is maximal in $X$.

(ii) Let $U$ be a maximal submodule of ${ }_{A} X$ and note that $U \subseteq(T U: T)_{X} \subseteq$ $X$. If $(T U: T)_{X}=X$, then $X=T X=T(T U: T)_{X} \subseteq T U$. That is, $U=$ $X$, a contradiction to the maximality of $U$. Thus $U=(T U: T)_{X}$. Now the fact that $T U$ is a maximal $T$-accessible submodule of $X$ follows easily from the correspondence and the maximality of $U$.

CoROllary 3.5. Let ${ }_{4} X$ be T-accessible. Then ${ }_{A} X$ has a maximal submodule if and only if ${ }_{A} X$ has a maximal $T$-accessible submodule. Furthermore, if $U$ is a maximal submodule of $X$, then $U$ contains a unique maximal T-accessible submodule $T U$ of $X$; and if $Y$ is a maximal T-accessible submodule of $X$, then $Y$ is contained in a unique maximal submodule $(Y: T)_{X}$ of $X$.

The next two lemmas follow from Theorems 3.4 and 3.2 respectively and are needed to answer our first question.

Lemma 3.6. For ${ }_{A} X$, let ${ }_{A} M$ be the intersection of all maximal $T$-accessible submodules of $T X$. Then $T M=T J(T X)$.

REMARK. If there are no maximal $T$-accessible submodules of $T X$, we set $M=T X$.

Proof. By Corollary 3.5 there are no maximal $T$-accessible submodules of $T X$ if and only if there are no maximal submodules of $T X$. In this case the lemma becomes trivial.

Thus let $\left\{H_{i}\right\}_{i \in I}$ be the set of all maximal submodules of $T X$. Then by Theorem $3.4\left\{T H_{i}\right\}_{i \in I}$ is the set of all maximal $T$-accessible submodules of $T X$. Clearly $M \subseteq J(T X)$ so that $T M \leqq T J(T X)$. But $T J(T X)=T\left(\bigcap_{i \in I} H_{i}\right) \subseteq \bigcap_{i \in I} T H_{i}=M$. Thus $T J(T X) \subseteq T M$.

Lemma 3.7. For ${ }_{A} X$, let ${ }_{A} M$ be defined as in Lemma 3.6 and let $\left\{L_{i}\right\}_{i \in I}$ be the collection of maximal submodules of ${ }_{B} \operatorname{Hom}_{A}\left(P^{*}, X\right)$. Then $P^{*}\left(\bigcap_{i \in I} L_{i}\right)=T M$.

Proof. By Theorem 3.2 there are no maximal $T$-accessible modules of $T X$ if and only if there are no maximal submodules of ${ }_{B} \mathrm{Hom}_{A}\left(P^{*}, X\right)$. 
In this case the lemma becomes trivial.

By the remarks following Theorem $3.2\left\{P^{*} L_{i}\right\}_{i \in I}$ is the set of all maximal $T$-accessible submodules of $T X$. Thus $P^{*}\left(\bigcap_{i \in I} L_{i}\right) \leqq$ $\bigcap_{i \in I} P^{*} L_{i}=M$, and so $P^{*}\left(\bigcap_{i \in I} L_{i}\right) \leqq T M$.

Conversely, $T M \subseteq P^{*} L_{i}$ for all $i \in I$. By Theorem $3.2 \mathrm{Hom}_{A}\left(P^{*}\right.$, $\left.P^{*} L_{i}\right)=L_{i}$, hence $\operatorname{Hom}_{A}\left(P^{*}, T M\right) \subseteq L_{i}$ for all $i \in I$. That is, $\operatorname{Hom}_{A}\left(P^{*}\right.$, $T M) \cong \bigcap_{i \in I} L_{i}$. Again by Theorem 3.2 TM=P* $\operatorname{Hom}_{A}\left(P^{*}, T M\right) \cong P^{*}$ $\left(\bigcap_{i \in I} L_{i}\right)$.

Theorem 3.8. Let $P_{A}$ be finitely generated projective with trace ideal $T$ and $B=$ End $\left(P_{A}\right)$. For ${ }_{A} X$, we have

(a) $P^{*} N\left(\operatorname{Hom}_{A}\left(P^{*}, X\right)\right)=T J(T X)$.

(b) $N\left(\operatorname{Hom}_{A}\left(P^{*}, X\right)\right)=\operatorname{Hom}_{A}\left(P^{*}, J(T X)\right)$.

Proof. (a) By Lemmas 3.7 and 3.6, $P^{*} N\left(\operatorname{Hom}_{A}\left(P^{*}, X\right)\right)=$ $P^{*}\left(\bigcap_{i \in I} L_{i}\right)=T M=T J(T X)$.

(b) By Theorem 3.2 and (a) we have that $N\left(\operatorname{Hom}_{A}\left(P^{*}, X\right)\right)=$ $\operatorname{Hom}_{A}\left(P^{*}, P^{*} N\left(\operatorname{Hom}_{A}\left(P^{*}, X\right)\right)=\operatorname{Hom}_{A}\left(P^{*}, T J(T X)\right)\right.$. But it is easy to see that $\operatorname{Hom}_{A}\left(P^{*}, T J(T X)\right)=\operatorname{Hom}_{A}\left(P^{*}, J(T X)\right)$.

Thus we have the following description of the radical of ${ }_{B} P \otimes{ }_{A} X$.

Corollary 3.9. (a) For ${ }_{A} X, N\left({ }_{B} P \otimes{ }_{A} X\right) \cong P \otimes_{A} J(T X)$.

(b) $N\left({ }_{B} P\right) \cong P \otimes{ }_{A} J(T)$.

Proof. (a) follows from Theorem 3.8 since $P \otimes_{A} X \cong \operatorname{Hom}_{A}\left(P^{*}, X\right)$ for all ${ }_{A} X$.

(b) follows in the same manner as (a) using ${ }_{B} P \cong{ }_{B} P \otimes{ }_{A} A$.

The following corollary is well known. See for example [11, Prop. 1.1].

Corollary 3.10. $N=N(B)=\operatorname{Hom}_{A}\left(P^{*}, J P^{*}\right)=\operatorname{Hom}_{A}(P, P J)$.

Proof. That $N=\operatorname{Hom}_{A}\left(P^{*}, J P^{*}\right)$ follows from the fact that $B=$ End $\left({ }_{A} P^{*}\right)$ and $T P^{*}=P^{*}$. That $N=\operatorname{Hom}_{A}(P, P J)$ follows by a dual argument.

Corollary 3.11. Letting $N=N(B)$, we have

(a) $P^{*} N=T J P^{*}$.

(b) $N P=P J T$.

Proof. (a) $P^{*} N=P^{*} \operatorname{Hom}_{A}\left(P^{*}, J P^{*}\right)=T J P^{*}$.

(b) follows by symmetry.

We now move on to our second question. For a right $A$-module $V_{A}$, Morita [8] has defined a right $A$-module $Y$ to be of $V$-dominant dimension $\geqq n$ (written $V$-dom. $\operatorname{dim} . \quad Y \geqq n$ ) if there is an exact 
sequence

$$
0 \longrightarrow Y \longrightarrow Y_{1} \longrightarrow \cdots \longrightarrow Y_{n}
$$

such that each $Y_{i}$ is a direct product of copies of $V$. Dually, for a left $A$-module ${ }_{A} U$, we say that a left $A$-module $X$ is of $U$-dominant codimension $\geqq n$ (written $U$-dom. codim. $X \geqq n$ ) if there is an exact sequence

$$
X_{n} \longrightarrow \cdots \longrightarrow X_{1} \longrightarrow X \longrightarrow 0
$$

such that each $X_{i}$ is a direct sum of copies of $U$.

Let $\mathfrak{D}_{n}\left(V_{A}\right)$ be the full subcategory of $\mathfrak{M}_{A}$ consisting of all $A$ modules of $V$-dominant dimension $\geqq n$. Similarly, let $\mathscr{C}_{n}\left({ }_{A} U\right)$ be the full subcategory of ${ }_{A} \mathfrak{M}$ consisting of all $A$-modules of $U$-dominant codimension $\geqq n$.

In particular $\mathscr{C}_{1}\left({ }_{A} P^{*}\right)$ consists of all modules ${ }_{A} X$ that are homomorphic images of a direct sum of copies of ${ }_{A} P^{*}$. By Lemma 3.1 we see that $\mathscr{C}_{1}\left({ }_{A} P^{*}\right)$ is the full subcategory of $T$-accessible left $A$-modules.

Let $W_{B}$ be an injective cogenerator in $\mathfrak{M}_{B}$. That is $W_{B}$ is injective and $\mathfrak{D}_{1}\left(W_{B}\right)=\mathfrak{M}_{B}$. Let $Q_{A}=\operatorname{Hom}_{B}\left({ }_{A} P_{B}^{*}, W_{B}\right)_{A}$. Then $Q_{A}$ is injective by [8, Lemma 1.3]. By the natural isomorphism (see I)

$$
\operatorname{Hom}_{A}\left(Y_{A}, \operatorname{Hom}_{B}\left({ }_{A} P_{B}^{*}, W_{B}\right)_{A}\right) \cong \operatorname{Hom}_{B}\left(Y \otimes{ }_{A} P_{B}^{*}, W_{B}\right)
$$

and the fact that $W_{B}$ is a cogenerator, we have

$$
Y \otimes{ }_{A} P^{*}=0 \text { if and only if } \operatorname{Hom}_{A}\left(Y_{A}, Q_{A}\right)=0 \text {. }
$$

LeMma 3.12. (a) ${ }_{A} X \in \mathscr{C}_{1}\left({ }_{A} P^{*}\right)$ if and only if $A / T \otimes{ }_{A} X=0$. (b) $Y_{A} \in \mathfrak{D}_{1}\left(Q_{A}\right)$ if and only if $\operatorname{Hom}_{A}(A / T, Y)=0$.

Proof. (a) Since $A / T \otimes{ }_{A} X \cong X / T X$, we have that $A / T \otimes X=$ 0 if and only if $X$ is $T$-accessible.

(b) Let $K=\bigcap\left\{\operatorname{ker} f \mid f \in \operatorname{Hom}_{A}(Y, Q)\right\}$. Since $Q_{A}$ is injective $\operatorname{Hom}_{A}(K$, $Q)=0$, which implies that $K \otimes{ }_{A} P^{*}=0$. Thus $K T=0$. That is, $K \subseteq l_{Y}(T)$. On the other hand, $l_{Y}(T) \otimes_{A} P^{*}=0$, so that $\operatorname{Hom}_{A}\left(l_{Y}(T), Q\right)=$ 0. Thus $l_{Y}(T) \cong K$. Therefore, $K=l_{Y}(T) \cong \operatorname{Hom}_{A}(A / T, Y)$.

If $Y \in \mathfrak{D}_{1}\left(Q_{A}\right)$, there is a monomorphism $\varphi: Y \rightarrow \Pi_{i \in I} Q^{(i)}$ where $Q^{(i)} \cong Q$ and $I$ is some index set. Let $\rho_{i}$ be the $i$ th projection map and let $f_{i}=\rho_{i} \varphi$. Then $\left.K \cong \bigcap_{i \in I} \operatorname{ker} f_{i}\right\}=0$.

Conversely, if $K=0$, let $I=\operatorname{Hom}_{A}(Y, Q)$ and define $\varphi: Y \rightarrow \Pi_{i \in I} Q^{(i)}$ by $\varphi(y)=(f(y))_{f \in I}$. Since $\operatorname{ker} \varphi=K=0, \varphi$ is monomorphism.

LEMMA 3.13.

(a) For $X \in \mathscr{C}_{1}\left({ }_{A} P^{*}\right), P / P J \otimes{ }_{A} X \cong P / N P \otimes{ }_{A} X$.

(b) For $Y \in \mathfrak{D}_{1}\left(Q_{A}\right), \mathrm{Hom}_{A}(P / P J, Y) \cong \mathrm{Hom}_{A}(P / N P, Y)$. 
Proof. By Corollary 3.11, NP=PJT. Thus we have the following exact sequence

$$
0 \longrightarrow \frac{P J}{P J T} \longrightarrow \frac{P}{N P} \longrightarrow \frac{P}{P J} \longrightarrow 0 \text {. }
$$

(a) For $X \in \mathscr{C}_{1}\left({ }_{A} P^{*}\right)$

$$
P J / P J T \otimes{ }_{A} X \longrightarrow P / N P \otimes{ }_{A} X \longrightarrow P / P J \otimes_{A} X \longrightarrow 0
$$

is exact. But $P J / P J T \otimes{ }_{A} X \cong P J \otimes A / T \otimes X=0$ by Lemma 3.12. (b) For $Y \in \mathfrak{D}_{1}\left(Q_{A}\right)$

$$
0 \longrightarrow \operatorname{Hom}_{A}(P / P J, Y) \longrightarrow \operatorname{Hom}_{A}(P / N P, Y) \longrightarrow \operatorname{Hom}_{A}(P J / P J T, Y)
$$

is exact. But $\operatorname{Hom}_{A}(P J / P J T, Y) \cong \operatorname{Hom}_{A}(P J \otimes A / T, Y) \cong \operatorname{Hom}_{A}(P J$, $\left.\operatorname{Hom}_{A}(A / T, Y)\right)=0$ by Lemma 3.12 .

A two-sided ideal $H$ of a ring $A$ is said to be left $T$-nilpotent if given any sequence $\left\{h_{i}\right\}_{i=1}^{\infty} \subseteq H$ there is a finite index $n$ such that $h_{1} h_{2} \cdots h_{n}=0$. We need the following necessary and sufficient conditions for a two-sided ideal $H$ to be left $T$-nilpotent.

Theorem 3.14. Let $H$ be a two-sided ideal of $A$. Then the following statements are equivalent.

(a) $H$ is left $T$-nilpotent.

(b) $\operatorname{Hom}_{A}\left(A / H, Y_{A}\right)=0$ implies $Y_{A}=0$.

(c) $A / H \otimes{ }_{A} X=0$ implies ${ }_{A} X=0$.

(d) For ${ }_{A} X \neq 0, H X^{\prime} \subseteq Y$.

Remark. Condition (b) says that if $Y_{A} \neq 0$, then $l_{Y}(H) \neq 0$. Condition (c) says that if ${ }_{A} X \neq 0$, then $H X \neq X$. The proof of (d) $\Rightarrow$ (a) is essentially in Bass [3] but will be included here for completeness.

Proof. (a) $\Rightarrow$ (b). Let $0 \neq y \in Y_{A}$. If $l_{Y}(H)=0$, then there exists $a_{1} \in H$ such that $y a_{1} \neq 0$. Again, there exists $a_{2} \in H$ such that $y a_{1} a_{2} \neq$ 0 . Clearly this will lead to a contradiction of (a) if $l_{Y}(H)=0$. Thus $l_{Y}(H) \neq 0$.

$(\mathrm{b}) \Rightarrow(\mathrm{c}) . \quad$ Suppose that $A / H \otimes{ }_{A} X=0$. Let $D=\operatorname{End}\left({ }_{A} X\right)$ and let $W_{D}$ be a cogenerator in $\mathfrak{M}_{D}$. Then

$$
0=\operatorname{Hom}_{D}\left(A / H \otimes{ }_{A} X_{D}, W_{D}\right) \cong \operatorname{Hom}_{A}\left(A / H, \operatorname{Hom}_{D}\left({ }_{A} X_{D}, W_{D}\right)\right) \text {. }
$$

Thus by $(\mathrm{b}) \operatorname{Hom}_{D}\left(X_{D}, W_{D}\right)=0$. Since $W_{D}$ is a cogenerator, $X_{D}=0$. Hence ${ }_{A} X=0$.

(c) $\Rightarrow(\mathrm{d})$. Let ${ }_{A} X \neq 0$ and suppose $H X$ is not small in $X$. Then there exists $K \varsubsetneqq X$ such that $H X+K=X$. However, for $\bar{X}=X / K$ we have $H \bar{X}=\bar{X}$. Thus by (c) $\bar{X}=0$, a contradiction. So $H X^{\prime} \subseteq X$. 
(d) $\Rightarrow$ (a). Let $\left\{h_{i}\right\}_{i=1}^{\infty} \subseteq H$ and let ${ }_{A} N$ be a free left $A$-module with basis $\left\{n_{i}\right\}_{i=1}^{\infty}$. Now $N=N_{1}+N_{2}$ where $N_{1}$ is the submodule of $N$ generated by $\left\{h_{i} n_{i+1}\right\}_{i=1}^{\infty}$ and $N_{2}$ is the submodule of $N$ generated by $\left\{n_{i}-h_{i} n_{i+1}\right\}_{i=1}^{\infty}$. Since $N_{1} \subseteq H N$ we have that $N=N_{2}$. So

$$
\begin{aligned}
n_{1} & =\sum_{i=1}^{t} a_{i}\left(n_{i}-h_{i} n_{i+1}\right) \\
& =a_{1} n_{1}+\sum_{i=2}^{t}\left(a_{i}-a_{i-1} h_{i-1}\right) n_{i}-a_{t} h_{t} n_{t+1} .
\end{aligned}
$$

Thus, by uniqueness of representation, $a_{1}=1$ and $0=a_{t} h_{t}=a_{t-1} h_{t-1} h_{t}=$ $\cdots=h_{1} h_{2} \cdots h_{t}$. Hence $H$ is left $T$-nilpotent.

For $P_{A}$ finitely generated projective we now give necessary and sufficient conditions for the radical $N$ of the endomorphism ring $B$ of $P_{A}$ to be left $T$-nilpotent in terms of the subcategories $\mathscr{C}_{1}\left({ }_{A} P^{*}\right)$ and $\mathfrak{D}_{1}\left(Q_{A}\right)$.

THEOREM 3.15. Let $P_{A}$ be finitely generated projective with trace ideal $T$ and $B=$ End $\left(P_{A}\right)$. Let $N$ be the radical of $B$. Then the following statements are equivalent.

(a) $N$ is left T-nilpotent.

(b) For $Y \in \mathfrak{D}_{1}\left(Q_{A}\right), \operatorname{Hom}_{A}\left(A / J, Y_{A}\right)=0$ implies $Y=0$.

(c) For $X \in \mathscr{C}_{1}\left({ }_{A} P^{*}\right), A / J \otimes{ }_{A} X=0$ implies $X=0$.

(d) JT is left T-nilpotent.

Proof. $(a) \Rightarrow(\mathrm{b})$. Let $Y \in \mathfrak{D}_{1}\left(Q_{A}\right)$. If $\operatorname{Hom}_{A}(A / J, Y)=0$, then by Lemma $3.13 \operatorname{Hom}_{A}\left(P, \operatorname{Hom}_{A}(A / J, Y)\right) \cong \operatorname{Hom}_{A}\left(P \otimes{ }_{A} A / J, Y\right) \cong$ $\operatorname{Hom}_{A}(P / P J, Y) \cong \operatorname{Hom}_{A}(P / N P, Y) \cong \operatorname{Hom}_{A}\left(B / N \otimes{ }_{B} P, Y\right) \cong \operatorname{Hom}_{B}(B / N$, $\left.\operatorname{Hom}_{A}(P, Y)\right)=0$. Thus $\operatorname{Hom}_{A}(P, Y)=0$ by Theorem 3.14. Hence $\operatorname{Hom}_{A}(Y, Q)=0$ which implies that $Y=0$ since $Y \in \mathfrak{D}_{1}\left(Q_{A}\right)$.

$(b) \Rightarrow(c)$. Let $X \in \mathscr{C}_{1}\left({ }_{A} P^{*}\right)$ and suppose $A / J \otimes{ }_{A} X=0$. Let $D=$ End $\left({ }_{A} X\right)$ and let $W_{D}$ be a cogenerator in $\mathfrak{M}_{D}$. We have $\mathrm{Hom}_{D}(A / J \otimes$ $\left.{ }_{A} X, W\right) \cong \operatorname{Hom}_{A}\left(A / J, \operatorname{Hom}_{D}(X, W)\right)=0$. By Lemma 3.12 $\operatorname{Hom}_{D}(X, W)_{A} \in$ $\mathfrak{D}_{1}\left(Q_{A}\right)$ since $\operatorname{Hom}_{A}\left(A / T, \operatorname{Hom}_{D}(X, W)\right) \cong \operatorname{Hom}_{D}\left(A / T \otimes_{A} X, W\right)=0$. Thus $\operatorname{Hom}_{D}(X, W)=0$ by (b). So $X_{D}=0$ since $W_{D}$ is a cogenerator. Thus ${ }_{A} X=0$.

(c) $\Rightarrow(\mathrm{d})$. For ${ }_{A} K$, suppose that $A / J T \otimes{ }_{A} K=0$; that is, $J T K=K$. Since $J T K \subseteq T K \subseteq K$, we see that $J T K=T K$, i.e., $A / J \otimes T K=0$. Thus $K=T K=0$ by (c). So $J T$ is left $T$-nilpotent by Theorem 3.14. $(\mathrm{d}) \Longrightarrow(\mathrm{c})$. Let $X \in \mathscr{C}_{1}\left({ }_{A} P^{*}\right)$ and suppose that $A / J \otimes{ }_{A} X=0$, i.e., $J X=$ $X$. Since $T X=X, J T X=X$. Hence $X=0$ by (d).

(c) $\Rightarrow$ (a). For ${ }_{B} U$, if $B / N \otimes_{B} U=0$, then $P^{*} \otimes_{B} B / N \otimes_{B} U \cong P^{*} / P^{*} N$ $\otimes_{B} U=0$. By Corollary 3.11

$$
0 \longrightarrow \frac{J P^{*}}{T J P^{*}} \longrightarrow \frac{P^{*}}{P^{*} N} \longrightarrow \frac{P^{*}}{J P^{*}} \longrightarrow 0
$$


is exact. Hence $P^{*} / J P^{*} \otimes_{B} U \cong A / J \otimes_{A} P^{*} \otimes_{B} U=0$. Thus $P^{*} \otimes_{B} U=$ 0 since $P^{*} \otimes_{B} U \in \mathscr{C}_{1}\left({ }_{A} P^{*}\right)$. But then ${ }_{B} U \cong P \otimes_{A} P^{*} \otimes_{B} U=0$.

If the radical $J$ of $A$ is left $T$-nilpotent, then (b) of Theorem 3.15 holds. Hence if $J$ is left $T$-nilpotent we have that $N$ is left $T$-nilpotent. This gives a functorial proof of a well-known result.

COROLlaRY 3.16. For $P_{A}$ finitely generated projective the following statements are equivalent.

(a) $N$ is left T-nilpotent.

(b) For $X \in \mathscr{C}_{1}\left({ }_{A} P^{*}\right), J X^{\prime} \subseteq X$.

(c) $J\left({ }_{A} P^{* I}\right)^{\prime} \subseteq{ }_{A} P^{* I}$ for any index set $I$.

REMARK. R. Ware [11, Lemma 5.3] has shown the equivalence of (a) and (c) in the case that $P_{A}$ is a projective module which is a finite direct sum of cyclic modules.

Proof. $\quad(a) \Rightarrow(b)$. Let ${ }_{A} X$ be $T$-accessible. If $J X$ is not small in $X$, there exists $H \varsubsetneqq X$ such that $J X+H=X$. However $\bar{X}=$ $X / H$ is also $T$-accessible, and $J \bar{X}=\bar{X}$. Thus by Theorem $3.15, \bar{X}=0$, a contradiction.

(b) $\Rightarrow$ (c). This is trivial since ${ }_{A} P^{* I} \in \mathscr{C}_{1}\left({ }_{A} P^{*}\right)$ for every index set $I$. (c) $\Rightarrow$ (b). Let $\alpha:{ }_{A} U \rightarrow{ }_{A} X$ be an $A$-epimorphism where $J U^{\prime} \subseteq U$. Then $X \cong U / K$ where $K=\operatorname{ker} \alpha$. Since $J[U / K]=(J U+K) / K$, if $(J U+K) / K+$ $H / K=U / K$, then $J U+K+H=U$. Thus $K+H=U$, which implies that $H=U$. Hence $J X^{\prime} \subseteq X$. (b) now follows from Lemma 3.1. (b) $\Rightarrow$ (a). Let $X \in \mathscr{C}_{1}\left({ }_{A} P^{*}\right)$ and suppose $J X=X$. Then $X=0$ since $J X^{\prime} \subseteq X$. Hence $N$ is left $T$-nilpotent by Theorem 3.15 .

4. Perfect injectors and perfect projectors. For $P_{A}$ finitely generated projective Anderson [1] has called $P_{A}$ a perfect injector (perfect projector) if the functor $F={ }_{B} P \otimes{ }_{A}($ ) preserves injective hulls (projective covers). Clearly a perfect injector (perfect projector) is also an injector (projector). Perfect injectors are characterized in terms of their trace ideal and certain conditions on large submodules.

LEMma 4.1. Let $I$ be a right ideal of $A$. Then $A / I$ is flat in $\mathfrak{M}_{A}$ if and only if $x \in I$ implies $x \in I x$.

Proof. Let $x \in I$ and consider the exact sequence

$$
0 \longrightarrow I \longrightarrow A \longrightarrow A / I \longrightarrow 0 \text {. }
$$

By [5, Proposition 2.2], assuming $(A / I)_{A}$ is flat, there exists a $\operatorname{map} \theta: A_{A} \rightarrow I_{A}$ such that $\theta(x)=x$. Thus $x=\theta(x)=\theta(1 x)=\theta(1) x \in I x$.

Conversely if $x \in I x$, then $x=i x$ for some $i \in I$. Define $\theta_{x}: A \rightarrow I$ 
by $\theta_{x}(a)=i a$ for $a \in A$. Then $\theta_{x}(x)=i x=x$. Again by [5, Proposition 2.2] we have that $(A / I)_{A}$ is flat.

THEOREM 4.2. For $P_{A}$ finitely generated projective the following statements are equivalent.

(a) $P_{A}$ is a perfect injector.

(b) $F={ }_{B} P \otimes{ }_{A}()$ preserves essential monomorphisms.

(c) $A / T$ is flat in $\mathfrak{M}_{A}$.

(d) The functor ${ }_{A} T \otimes{ }_{A}($ ) preserves essential monomorphisms.

(e) ${ }_{A} X \subseteq{ }_{A} E$ implies $T X \subseteq \subseteq^{\prime} T E$.

REMARK. The equivalence of (a), (b), and (c) is essentially (using Lemma 4.1) Anderson's result [1, Theorem 2.4].

Proof. $\quad(\mathrm{c}) \Leftrightarrow(\mathrm{e})$. Let ${ }_{A} X \subseteq{ }_{A} E$ and suppose $(A / T)_{A}$ is flat. Tensoring with $(A / T)_{A}$ we get the exact sequence

$$
0 \longrightarrow X / T X \longrightarrow E / T E \text {. }
$$

Therefore, $X \cap T E=T X$. Let $0 \neq K \subseteq T E \subseteq E$. Then $K \cap T X=$ $K \cap T E \cap X=K \cap X \neq 0$ since $X \subseteq^{\prime} E$. Thus $T X \subseteq^{\prime} T E$.

Conversely, by Lemma 4.1, to show $(A / T)_{A}$ is flat, it is sufficient to show $x \in T$ implies $x \in T x$. If $x \notin T x$, by Zorn's Lemma choose ${ }_{A} I \subseteq{ }_{A} A$ maximal with respect to the property that $T x \leqq I$, but $x \notin I$. Then $(A x+I) / I \subseteq^{\prime} A / I$, hence by assumption $T((A x+I) / I) \subseteq^{\prime} T(A / I)$. Since $T((A x+I) / I)=0$, we must have $T(A / I)=(T+I) / I=0$. That is, $T \subseteq I$. This is a contradiction since $x \in T$.

(c) $\Rightarrow(\mathrm{d})$. Let $\alpha:{ }_{A} X \rightarrow{ }_{A} E$ be an essential monomorphism. Clearly we may assume $\alpha$ is the inclusion map. Since $(A / T)_{A}$ is fiat, so is $T_{A}$, and thus $T \otimes{ }_{A} X \stackrel{1 \otimes \alpha}{\longrightarrow} T \otimes{ }_{A} E$ is one-to-one. Again since $(A / T)_{A}$ is flat, $T \otimes X \cong T X$ and $T \otimes E \cong T E$. Hence (d) follows by (e).

$(d) \Rightarrow(c)$. The proof of $(d) \Rightarrow(c)$ is almost identical (with the obvious changes) to the proof of $(\mathrm{e}) \Rightarrow(\mathrm{c})$, and hence will be omitted.

The following corollary indicates when $T$ is a direct summand of A.

COROLlary 4.3. For $P_{A}$ finitely generated projective the following statements are equivalent.

(a) $P_{A}$ is a perfect injector and $T_{A}$ is finitely generated.

(b) $T_{A}=e A$ for some idempotent $e \in A$.

REMARK. Corollary 4.3 generalizes a result of Anderson's [1, Corollary 2.7] that over a right perfect ring $A, P_{A}$ is a perfect injector if and only if $T_{A}$ is a direct summand of $A_{A}$. 
Proof. $\quad(a) \Rightarrow(b)$. By Theorem 4.2 we have that $(A / T)_{A}$ is flat. Since $T_{A}$ is finitely generated, $(A / T)_{A}$ is projective by [5, Corollary to Proposition 2.2]. Therefore $T_{A}$ is a direct summand of $A_{A}$. (b) $\Rightarrow\left(\right.$ a). Clearly $(A / T)_{A}$ is flat and $T_{A}$ is finitely generated. Thus (a) follows by Theorem 4.2.

We now give an example of a perfect injector whose trace is not finitely generated.

ExAmple 4.4. Let $A=\prod_{i \in I} K_{i}$ where $K_{i}=K$ a field and the index set $I$ is infinite. Let $T=K_{i}^{I}$. We may write $T=\left(e_{i} A\right)^{I}$ where $e_{i}^{2}=e_{i} \in A$ and $K_{i} \cong e_{i} A$. Let $P_{A}=e_{1} A$. Then $P_{A}$ is finitely generated projective and the trace ideal of $P_{A}$ is $T . \quad(A / T)_{A}$ is flat since $A$ is a regular ring, hence $P_{A}$ is a perfect injector. However, $T_{A}$ is not finitely generated.

Ideally we would like to give a characterization of perfect projectors dual to that of Theorem 4.2. In general we do not know enough pertinent information about small submodules to obtain an exact dualization. Anderson circumvented this problem by assuming that the ring $A$ is left perfect. He gives the following theorem.

TheOREM 4.5. (Anderson [1, Theorem 3.3]). Let $A$ be left perfect and let $P_{A}$ be finitely generated projective. Then the following statements are equivalent.

(a) $P_{A}$ is a perfect projector.

(b) $F={ }_{B} P \otimes_{A}($ ) preserves minimal epimorphisms.

(c) $J T=J \cap T$.

(d) ${ }_{A}(A / T)$ is projective in ${ }_{A} \mathfrak{M}$.

Our purpose in this discussion is to extend Anderson's result by replacing the condition that $A$ be left perfect with substantially weaker conditions on $P_{A}$.

Lemma 4.6. For ${ }_{A} K \subseteq_{A} X$, the following statements are equivalent.

(a) ${ }_{B} P \otimes{ }_{A} K^{\prime} \cong{ }_{B} P \otimes{ }_{A} X$.

(b) $T K^{\prime} \leqq T X$.

Proof. (a) $\Rightarrow(\mathrm{b})$. Suppose that $T K+H=T X$. Since $F$ is an exact additive functor, we may write $F(T K+H)=F(T K)+F(H)=$ $F(K)+F(H)=F(T X)=F(X)$. Thus $F(H)=F(X)$ which implies that $T H=T X$. Hence $H=T X$ since $T H \subseteq H \subseteq T X$.

(b) $\Rightarrow$ (a). Suppose that $F(K)+L=F(X)$. Then $P^{*} F(K)+P^{*} L=$ $T K+P^{*} L=P^{*} F(X)=T X$. Thus $P^{*} L=T X$ which implies that $L=F\left(P^{*} L\right)=F(T X)=F(X)$. 
CoROLlary 4.7. The functor $F={ }_{B} P \otimes{ }_{A}()$ always preserves projective covers of $T$-accessible left $A$-modules.

Proof. Let ${ }_{A} Y$ be $T$-accessible and let $\alpha:{ }_{A} X \rightarrow{ }_{A} Y$ be a projective cover of ${ }_{A} Y$. We have the following commutative diagram

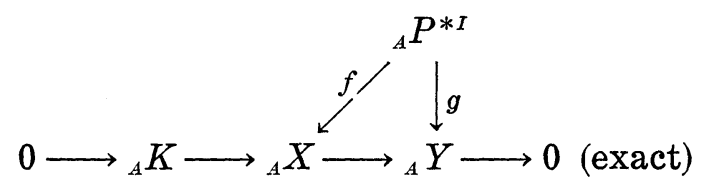

where $K=\operatorname{ker} \alpha, g$ follows by Lemma 3.1, and $f$ follows by the projectivity of ${ }_{A} P^{* I}$. Since $K^{\prime} \subseteq X, f$ is onto. Thus ${ }_{A} X$ is $T$-accessible, so $T K^{\prime} \subseteq X=T X$. Therefore, $F(\alpha): F(X) \rightarrow F(Y)$ is a minimal epimorphism by Lemma 4.6 .

Since ${ }_{A} X$ is projective

$$
{ }_{A} P^{* I} \longrightarrow{ }_{A} X \longrightarrow 0
$$

is split exact. Thus

$$
F\left(P^{* I}\right) \longrightarrow F(X) \longrightarrow 0
$$

is also split exact. Hence $F(X)$ is projective since it is a direct summand of $F\left(P^{* I}\right) \cong{ }_{B} B^{I}$.

The following result follows easily from Theorem 2.8 and Lemma 4.6, hence the proof will be omitted.

THEOREM 4.8. For $P_{A}$ finitely generated projective the following statements are equivalent.

(a) $P_{A}$ is a perfect projector.

(b) ${ }_{B} P$ is projective and for ${ }_{A} X$ projective, ${ }_{A} K^{\prime} \subseteq{ }_{A} X$ implies $T K^{\prime} \subseteq T X$.

THEOREM 4.9. Let $P_{A}$ be finitely generated projective with trace ideal $T$ and $B=$ End $\left(P_{A}\right)$. Let the radical $N$ of $B$ (equivalently $J T$ ) be left T-nilpotent. Then the following statements are equivalent.

(a) $P_{A}$ is a perfect projector.

(b) ${ }_{B} P$ is projective and $J T=J \cap T$.

(c) ${ }_{B} P$ is projective and $T J \subseteq J T$.

(d) ${ }_{B} P$ is projective and $P J=N P$.

REMARK. The proof that $J T=J \cap T$ in (a) $\Rightarrow(\mathrm{b})$ is due to Anderson [1].

Proof. $\quad(\mathrm{a}) \Rightarrow(\mathrm{b}) . \quad$ By Theorem $2.8,{ }_{B} P$ is projective and $\eta_{A}:{ }_{A} P^{*} \otimes$ ${ }_{B} P \rightarrow{ }_{A} T$ is a projective cover. Hence $J(T)=J T$ by Lemma 1.1. 
Clearly $J T \subseteq J \cap T$. Let $x \in J \cap T$ and suppose that $x \notin J T=J(T)$. Then there is a maximal submodule $H$ of $T$ such that $x \notin H$. Since $x \in J$, the natural map $A \longrightarrow A / A x$ is a projective cover. By (a) $P \rightarrow$ $P / P x$ is a projective cover, hence ${ }_{B} P x^{\prime} \subseteq{ }_{B} P$. But $P=P T=P H+P x$, since $T=H+A x$. Thus $P=P H$, and so $P^{*} \otimes P=P^{*} \otimes P H$. Hence $T=T H \subseteq H$, a contradiction. Thus $J T=J \cap T$.

(b) $\Rightarrow$ (c). Clearly $T J \subseteq J \cap T$. By assumption $J \cap T=J T$. Thus $T J \subseteq J T$.

$(\mathrm{c}) \Rightarrow(\mathrm{d})$. Clearly $P J T \subseteq P J$. Since $T J \subseteq J T$ we have that $P J=$ $P T J \subseteq P J T$. Thus $P J=P J T$. Hence $P J=N P$ by Corollary 3.11 . $(\mathrm{d}) \Rightarrow(\mathrm{a})$. Let $\alpha:{ }_{A} X \rightarrow{ }_{A} Y$ be a projective cover. ${ }_{B} P \otimes{ }_{A} X$ is projective since $P_{A}$ is a projector. Let $K=\operatorname{ker} \alpha$. Since $K^{\prime} \subseteq X, K \subseteq$ $J(X)=J X$. Since $P_{A}$ and ${ }_{A} X$ are flat, both $P \otimes J X$ and $P J \otimes X=$ $N P \otimes X$ have the same image in $P \otimes X$ under the maps $P \otimes J X \rightarrow$ $P \otimes X$ and $P J \otimes X \rightarrow P \otimes X$ respectively. Thus $P \otimes K \subseteq N P \otimes X$. Hence $P \otimes K^{\prime} \subseteq P \otimes X$ because $N$ is left $T$-nilpotent. Therefore $P \otimes$ $X \stackrel{1 \otimes \alpha}{\longrightarrow} P \otimes Y$ is a projective cover.

To suppose $N$ is left $T$-nilpotent is one way to weaken Anderson's condition that $A$ be left perfect. Another way is as follows. Instead of assuming that every left $A$-module has a projective cover, we assume that a particular left $A$-module has a projective cover. But first we need the following lemma.

Lemma 4.10. ${ }_{A}(A / T)$ is projective in ${ }_{A} \mathfrak{M}$ if and only if $A=$ $T+r_{A}(T)$.

Proof. If ${ }_{A}(A / T)$ is projective then ${ }_{A} T$ is a direct summand of ${ }_{A} A$. Hence ${ }_{A} A={ }_{A} T+{ }_{A} U$. Clearly ${ }_{A} U \subseteq r_{A}(T)$. Thus $A=T+r_{A}(T)$. Conversely, if $A=T+r_{A}(T)$, then $1=t_{0}+u$ where $t_{0} \in T, u \in$ $r_{A}(T)$. If $t \in T$, then $t=t 1=t t_{0}+t u=t t_{0}$. That is, $T=A t_{0}$; hence ${ }_{A} T$ is finitely generated. Furthermore, for $t \in T$ we see that $t=t t_{0} \in$ $t T$. Hence by Lemma 4.1, ${ }_{A}(A / T)$ is flat. Thus ${ }_{A}(A / T)$ is projective [5, Corollary to Proposition 2.2].

THEOREM 4.11. Let $P_{A}$ be finitely generated projective with trace ideal $T$ and $B=$ End $\left(P_{A}\right)$. Then the following statements are equivalent.

(a) $P_{A}$ is a perfect projector and ${ }_{A}(A / T)$ has a projctive cover.

(b) $F={ }_{B} P \otimes{ }_{A}\left(\right.$ ) preserves minimal epimorphisms and ${ }_{A}(A / T)$ has a projective cover.

(c) ${ }_{A}(A / T)$ is projective in ${ }_{A} \mathfrak{M}$.

Proof. $\quad(\mathrm{a}) \Longrightarrow(\mathrm{c})$. Let $\alpha:{ }_{A} X \rightarrow{ }_{A}(A / T)$ be a projective cover of ${ }_{A}(A / T)$ and let $K=\operatorname{ker} \alpha$. Then $F(\alpha): F(X) \rightarrow F(A / T)$ is a projective 
cover. However, $F(A / T)=0$ which implies that $F(X)=0$ since ker $F(\alpha)^{\prime} \subseteq F(X)$. Hence $F(K)=0$, so $T K=0$.

By the projectivity of ${ }_{A} A$ we have the following commutative diagram.

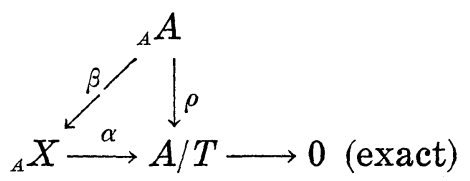

where $\rho$ is the natural map. $\beta$ is onto since $K^{\prime} \leqq X$. Now $K=$ $(T) \beta=T(T) \beta=T K=0$. Thus $\alpha$ is an isomorphism, hence ${ }_{A}(A / T)$ is projective.

(c) $\Rightarrow$ (a). Let $\alpha:{ }_{A} X \rightarrow{ }_{A} Y$ be a projective cover and let $K=\operatorname{ker} \alpha$. Since ${ }_{A}(A / T)$ is projective, so is ${ }_{A} T$. Hence $P_{A}$ is a projector by Theorem 2.9. Thus ${ }_{B} P \otimes_{A} X$ is projective.

By Lemma 4.10, $A=T+r_{A}(T)$. Hence $X=A X=T X+r_{A}(T) X$. Suppose that $T K+H=T X$. Then $T K+H+r_{A}(T) X=T X+r_{A}(T) X=$ $X$. Thus $H+r_{A}(T) X=X$ since $T K \leqq K^{\prime} \leqq X$. Now $T H+\operatorname{Tr}_{A}(T) X=$ $T X$, and so $T H=T X$. Therefore, $H=T X$, so that $T K^{\prime} \subseteq T X$. Hence $F(\alpha): F(X) \rightarrow F(Y)$ is a projective cover by Lemma 4.6. $(b) \Leftrightarrow(c)$. This is essentially the proof of $(a) \Leftrightarrow(c)$.

CoROLlary 4.12. Let $A$ be semiperfect. Then the following statements are eqivalent.

(a) $P_{A}$ is a perfect projector.

(b) ${ }_{A} P^{*}$ is a perfect injector.

(c) ${ }_{A}(A / T)$ is projective in ${ }_{A} \mathfrak{M}$.

Proof. $\quad(a) \Rightarrow(b)$. Since $A$ is semiperfect, ${ }_{A}(A / T)$ has a projective cover. Thus ${ }_{A}(A / T)$ projective (hence flat) by Theorem 4.11. So ${ }_{A} P^{*}$ is a perfect injector by Theorem 4.2 .

(b) $\Rightarrow(\mathrm{c}) . \quad{ }_{A}(A / T)$ is flat by Theorem 4.2. Since $A$ is semiperfect, ${ }_{A}(A / T)$ has a projective cover. Thus ${ }_{A}(A / T)$ is projective by Lemma 1.2.

(c) $\Rightarrow$ (a). This follows easily by Theorem 4.11 .

EXAMPLE 4.13. We give an example to show that the condition that ${ }_{A}(A / T)$ have a projective cover is not redundant.

Let $F$ be a field, ${ }_{F} V$ an infinite dimensional vector space over $F$, and $A$ the ring of all linear transformations of ${ }_{F} V$. Since ${ }_{F} V$ is a generator, $V_{A}$ is finitely generated projective and $F=$ End $\left(V_{A}\right)$ (see [2]). Let $e:{ }_{F} V \rightarrow F v$ be the projection map onto the one-dimensional subspace $F v$ of ${ }_{F} V$. Clearly $V_{A} \cong e A$; hence, the trace ideal of $V_{A}$ is $T=$ AeA. Properties of $A,{ }_{F} V_{A}$, and $T$. 
(a) $T=$ Socle $\left(A_{A}\right)$ (see [6]).

(b) ${ }_{A}(A / T)$ is not projective since ${ }_{A} T$ is not a direct summand of ${ }_{A} A$, as ${ }_{A} T$ is not finitely generated.

(c) For ${ }_{A} X, \operatorname{Hom}_{A}(X, A)=0$ if and only if $V \otimes{ }_{A} X=0$.

(d) ${ }_{A} A$ is self-injective (e.g. see [9]).

(e) ${ }_{F} V \otimes{ }_{A}$ ( ) preserves minimal epimorphisms.

(f) $V_{A}$ is a projector, hence a perfect projector by (e).

Proof. (c) follows by the isomorphism

$$
\begin{aligned}
\operatorname{Hom}_{A}(X, A) & =\operatorname{Hom}_{A}\left(X, \operatorname{Hom}_{F}(V, V)\right) \\
& \cong \operatorname{Hom}_{F}\left(V \otimes{ }_{A} X, V\right) .
\end{aligned}
$$

(e). Let $\alpha:{ }_{A} X \rightarrow{ }_{A} Y$ be a minimal epimorphism with $K=\operatorname{ker} \alpha$. Then

$$
0 \longrightarrow V \otimes{ }_{A} K \longrightarrow V \otimes_{A} X \longrightarrow V \otimes_{A} Y \longrightarrow 0
$$

is an exact sequence. But $V \otimes{ }_{A} K=0$ since $\operatorname{Hom}_{A}(X, A)=0$. That is, given $f: K \rightarrow A$, then $f$ extends to $\hat{f}: X \rightarrow A$ by the self-injectivity of ${ }_{A} A$. Now $\hat{f}(K)^{\prime} \subseteq A$ which implies $\hat{f}(K)=0$, as $J(A)=0$. Hence $f=0$. (f). By Theorem 2.8, $V_{A}$ is a projector since ${ }_{F} V$ is projective.

\section{REFERENCES}

1. F. Anderson, Endomorphism rings projective modules, Math. Z., 111 (1969), 322-332.

2. G. Azumaya, Completely faithful modules and self-injective rings, Nagoya Math. J., 27 (1966), 697-708.

3. H. Bass, Finitistic dimension and a homological generalization of semi-primary rings, Trans. Amer. Math. Soc., 95 (1960), 466-488.

4. H. Cartan, and S. Eilenberg, Homological Algebra, Princeton Univ. Press, Princeton, N. J., 1956.

5. S. U. Chase, Direct products of modules, Trans. Amer. Math. Soc., 97 (1960), 457-473.

6. N. Jacobson, Structure of Rings, Amer. Math. Soc. Colloq. Pub., Vol. XXXVII, 1956.

7. J. Lambek, Lectures on Rings and Modules, Waltham, Mass., 1966.

8. K. Morita, Localizations in categories of modules. I., Math. Z., 114 (1970), 121-144.

9. F. Sandomierski, Some examples of right self-injective rings which are not left selfinjective, Proc. Amer. Math. Soc., 26, No. 2 (1970), 244-245.

10. Modules over the endomorphism ring of a finitely generated projective module, Proc. Amer. Math. Soc., 31 (1972), 27-31.

11. R. Ware, Endomorphism rings of projective modules, Trans. Amer. Math. Soc., 115 (1971), 233-256.

Received January 7, 1972. This paper was taken from the author's doctoral dissertation, under the direction of Professor F. L. Sandomierski, at the University of Wisconsin. The author expresses his gratitude to Professor Sandomierski for his guidance and encouragement. The author also thanks Professor Darrell R. Turnidge for his valuable assistance.

\section{Kent State UnIVERSity}

Current address: College of William and Mary

Williamsburg, Virginia 


\section{PACIFIC JOURNAL OF MATHEMATICS}

\section{EDITORS}

D. Gilbarg and J. Milgram

Stanford University

Stanford, California 94305

R. A. Beaumont

University of Washington

Seattle, Washington 98105
J. DUGUNDJI* Department of Mathematics

University of Southern California Los Angeles, California 90007

RICHARD ARENS

University of California

Los Angeles, California 90024

\section{ASSOCIATE EDITORS}

E. F. BeCKenbach

B. H. NeUMaNN

F. WOLF

K. Yoshida

\section{SUPPORTING INSTITUTIONS}

UNIVERSITY OF BRITISH COLUMBIA

UNIVERSITY OF SOUTHERN CALIFORNIA

CALIFORNIA INSTITUTE OF TECHNOLOGY

UNIVERSITY OF CALIFORNIA

MONTANA STATE UNIVERSITY

STANFORD UNIVERSITY

UNIVERSITY OF TOKYO

UNIVERSITY OF NEVADA

UNIVERSITY OF UTAH

NEW MEXICO STATE UNIVERSITY

WASHINGTON STATE UNIVERSITY

OREGON STATE UNIVERSITY

UNIVERSITY OF OREGON

OSAKA UNIVERSITY

UNIVERSITY OF WASHINGTON

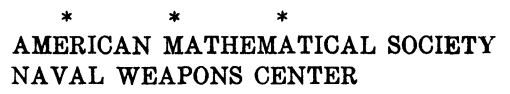

* C. DePrima will replace J. Dugundji until August 1974. 


\section{Pacific Journal of Mathematics}

\section{Vol. 47, No. $1 \quad$ January, 1973}

K. Adachi, Masuo Suzuki and M. Yoshida, Continuation of holomorphic

mappings, with values in a complex Lie group ....................

Michael Aschbacher, A characterization of the unitary and symplectic groups

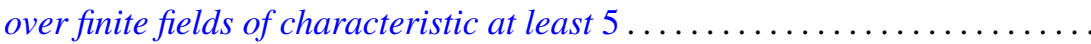

Larry Eugene Bobisud and James Calvert, Energy bounds and virial theorems for abstract wave equations....................................

Christer Borell, A note on an inequality for rearrangements ................

Peter Southcott Bullen and S. N. Mukhopadhyay, Peano derivatives and general

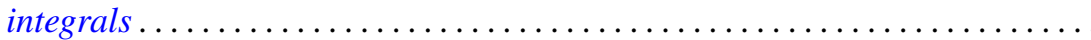

Wendell Dan Curtis, Yu-Lee Lee and Forrest Miller, A class of infinite dimensional subgroups of $\operatorname{Diff}^{r}(X)$ which are Banach Lie groups .........

Paul C. Eklof, The structure of ultraproducts of abelian groups ...............

William Alan Feldman, Axioms of countability and the algebra $C(X) \ldots \ldots \ldots$

Jack Tilden Goodykoontz, Jr., Aposyndetic properties of hyperspaces...........

George Grätzer and J. Płonka, On the number of polynomials of an idempotent algebra. II ...........................................

Alan Trinler Huckleberry, The weak envelope of holomorphy for algebras of

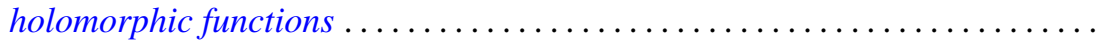

John Joseph Hutchinson and Julius Martin Zelmanowitz, Subdirect sum decompositions of endomorphism rings . . . . . . . . . . . . . . . .

Gary Douglas Jones, An asymptotic property of solutions of

$y^{\prime \prime \prime}+p y^{\prime}+q y=0$.

Howard E. Lacey, On the classification of Lindenstrauss spaces .

Charles Dwight Lahr, Approximate identities for convolution measure algebras.

George William Luna, Subdifferentials of convex functions on Banach

spaces.

Nelson Groh Markley, Locally circular minimal sets. .

Robert Wilmer Miller, Endomorphism rings of finitely generated projective modules

Donald Steven Passman, On the semisimplicity of group rings of linear

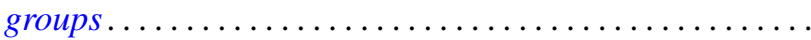

Bennie Jake Pearson, Dendritic compactifications of certain dendritic spaces.

Ryōtarō Satō, Abel-ergodic theorems for subsequences ...... .

Henry S. Sharp, Jr., Locally complete graphs. . .

Harris Samuel Shultz, A very weak topology for the Mikusinski field of

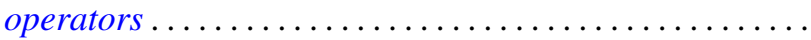

Elena Stroescu, Isometric dilations of contractions on Banach spaces ...

Charles W. Trigg, Versum sequences in the binary system ... . .

William L. Voxman, On the countable union of cellular decompositions of n-manifolds 